\title{
BILINEAR OPTIMAL CONTROL OF THE VELOCITY TERM IN A VON KÁRMÁN PLATE EQUATION
}

\author{
JONG YEOUL PARK ${ }^{1}$, SUN HYE PARK ${ }^{\bowtie 1}$ and YONG HAN KANG ${ }^{2}$
}

(Received 6 November, 2008; revised 8 April, 2009; first published online 29 July, 2013)

\begin{abstract}
We consider a bilinear optimal control problem for a von Kármán plate equation. The control is a function of the spatial variables and acts as a multiplier of the velocity term. We first state the existence of solutions for the von Kármán equation and then derive optimality conditions for a given objective functional. Finally, we show the uniqueness of the optimal control.
\end{abstract}

2010 Mathematics subject classification: 49J20.

Keywords and phrases: Kármán system, existence of solutions, optimal control, necessary conditions.

\section{Introduction}

We consider the following von Kármán plate equation:

$$
\begin{aligned}
& w_{t t}+\Delta^{2} w+b(x, y) w_{t}=\alpha[w, F(w)] \quad \text { in } Q=\Omega \times(0, T), \\
& w(\cdot, 0)=w_{0}, \quad w_{t}(\cdot, 0)=w_{1} \quad \text { in } \Omega, \\
& w=\frac{\partial w}{\partial v}=0 \quad \text { on } \Sigma_{0}=\Gamma_{0} \times(0, T), \\
& \Delta w+(1-\mu) B_{1} w=0 \quad \text { on } \Sigma_{1}=\Gamma_{1} \times(0, T), \\
& \frac{\partial}{\partial v}(\Delta w)+(1-\mu) B_{2} w=0 \quad \text { on } \Sigma_{1}=\Gamma_{1} \times(0, T),
\end{aligned}
$$

where $\Omega \subset \mathbb{R}^{2}, \Omega \ni(x, y)$, is a bounded domain with sufficiently smooth boundary $\partial \Omega ; \Gamma_{0} \cup \Gamma_{1}=\partial \Omega, \Gamma_{0} \cap \Gamma_{1}=\emptyset, \Gamma_{0}$ and $\Gamma_{1}$ have positive measures; $v=\left(v_{1}, v_{2}\right)$ is the outward unit normal vector on $\partial \Omega$; the constant $\mu, 0<\mu<1 / 2$, represents Poisson's

\footnotetext{
${ }^{1}$ Department of Mathematics, Pusan National University, Busan 609-735, South Korea; e-mail: sh-park@pusan.ac.kr.

${ }^{2}$ Institute of Liberal Education, Catholic University of Daegu, Gyeongsan 712-702, South Korea.

(c) Australian Mathematical Society 2013, Serial-fee code 1446-1811/2013 \$16.00
} 
ratio; and

$$
\begin{aligned}
& B_{1} w=2 v_{1} v_{2} w_{x y}-v_{1}^{2} w_{y y}-v_{2}^{2} w_{x x} \\
& B_{2} w=\frac{\partial}{\partial \tau}\left[\left(v_{1}^{2}-v_{2}^{2}\right) w_{x y}+v_{1} v_{2}\left(w_{y y}-w_{x x}\right)\right]
\end{aligned}
$$

The Airy stress function $F(w)$ satisfies the elliptic problem

$$
\Delta^{2} F(w)=-[w, w] \quad \text { in } \Omega, \quad F(w)=\frac{\partial}{\partial v} F(w)=0 \quad \text { on } \Gamma=\partial \Omega,
$$

where the von Kármán bracket $[w, \phi]$ is given by

$$
[w, \phi] \equiv w_{x x} \phi_{y y}+w_{y y} \phi_{x x}-2 w_{x y} \phi_{x y}
$$

Note that the von Kármán bracket is bilinear and symmetric. We take as our objective functional

$$
J(w, b)=\frac{1}{2} \int_{Q}(w-z)^{2} d Q+\frac{\beta}{2} \int_{\Omega} b(x, y)^{2} d \Omega,
$$

where $z \in L^{2}(Q)$ is the desired evolution for the plate, the quadratic term in $b$ represents the cost of implementing the control belonging in

$$
U_{M}=\left\{b \in L^{\infty}(\Omega):-M \leq b(x, y) \leq M\right\}, \quad M>0,
$$

with weighting factor $\beta>0$, and $w$ is a solution of (1.1)-(1.5) with respect to a given control $b(x, y)$. It is easily shown that $U_{M}$ is a closed and convex subset of $L^{\infty}(\Omega)$. For convenience, we assume that $z \in C\left([0, T] ; L^{2}(\Omega)\right)$ and $z_{t} \in C\left([0, T] ; L^{2}(\Omega)\right)$.

We consider the optimal control problem

$$
\operatorname{minimize}\left\{J(w, b): b \in U_{M}\right\} \text {. }
$$

Our purpose is to give some existence results of solutions for the von Kármán plate system (1.1)-(1.5) with $\alpha=1$, seek an optimal control $b^{*} \in U_{M}$ satisfying the optimal control problem (1.6), and derive some necessary optimality conditions for the optimal control pair $\left(w^{*}, b^{*}\right)$, where $w^{*}$ is the solution of (1.1)-(1.5) with respect to an optimal control $b^{*}$. From the physical point of view, $w$ in equation (1.1) denotes the transversal displacement and the Airy stress function $F(w)$ a vibrating plate.

Recently, some authors have studied a variety of von Kármán models [6-9, 11, 14, 15]. Favini et al. [8] proved global existence and uniqueness of weak solutions as well as the regularity of solutions to (1.1)-(1.2) for $b(x, y) \equiv 0$ with nonlinear boundary dissipation by using 'sharp' regularity of the Airy stress function (see Lemma 2.1). This regularity is critical to prove the uniqueness of weak solutions. Indeed, the standard regularity result $[10,12]$ says that if $w \in H^{2}(\Omega)$ then $F(w) \in H^{3-\epsilon}(\epsilon>0)$. This regularity does not imply that $F(w) \in W^{2, \infty}(\Omega)$. Thus, the uniqueness of weak solutions for von Kármán systems had been an open problem until sharp regularity $[6,8,9]$ was 
proved. Horn and Lasiecka [9] investigated asymptotic behaviour of weak solutions to a von Kármán model (1.1)-(1.2) with nonlinear boundary dissipation. Park and Park [14] proved the existence of solutions and uniform decay for a von Kármán plate equation with a boundary memory condition.

On the other hand, bilinear control problems were first studied by Bradley et al. $[4,5]$. Bradley and Lenhart [4] considered a bilinear spatial control problem (1.6) subject to Kirchhoff plate equations (1.1)-(1.5) with $\alpha=0$, and then in collaboration with Yong [5] treated the case of $b(t) w_{t}$ in equation (1.1) with $\alpha=0$. Motivated by their results, in this paper we consider a bilinear spatial control problem (1.6) with respect to a von Kármán plate system (1.1)-(1.5). It is a feature of our paper that the differentiability of the Airy stress function $F(\cdot)$ is established and the adjoint equations for von Kármán equations of the form (1.1)-(1.5) are introduced. It is very important to observe that as far as we know, few optimal control problems for von Kármán models have been considered in the literature as in the present paper.

The paper is organized as follows. We introduce the relevant results for the Airy stress function, von Kármán bracket and the existence of a weak solution to (1.1)-(1.5) in Section 2. We prove the existence of an optimal control pair for (1.6) in Section 3, and then derive the necessary optimality conditions and prove the uniqueness of the optimal control in Section 4 by following similar ideas to Bradley et al. [4, 5].

\section{Existence of a weak solution}

Throughout the paper we denote

$$
\begin{array}{ll}
W=\left\{w \in H^{2}(\Omega): w=\frac{\partial w}{\partial v}=0 \text { on } \Gamma_{0}\right\}, & \widetilde{W}=\left\{w \in H^{2}(\Omega): w=\frac{\partial w}{\partial v}=0 \text { on } \Gamma_{1}\right\}, \\
(w, \phi)=\int_{\Omega} w(x, y) \phi(x, y) d \Omega, & \|w\|_{L^{2}(\Omega)}^{2}=\int_{\Omega} w(x, y)^{2} d \Omega .
\end{array}
$$

Here $\|\cdot\|_{X}$ denotes the norm on a Banach space $X$. For simplicity, we denote $\|\cdot\|_{L^{2}(\Omega)}$ by $\|\cdot\|$. Define the bilinear symmetric form

$$
\begin{aligned}
a(w, \phi) & =\int_{\Omega}\left[\Delta w \Delta \phi+(1-\mu)\left(2 w_{x y} \phi_{x y}-w_{x x} \phi_{y y}-w_{y y} \phi_{x x}\right)\right] d \Omega \\
& =\int_{\Omega}\left[w_{x x} \phi_{x x}+w_{y y} \phi_{y y}+\mu\left(w_{x x} \phi_{y y}+w_{y y} \phi_{x x}\right)+2(1-\mu) w_{x y} \phi_{x y}\right] d \Omega .
\end{aligned}
$$

We know [10] that $a(w, w)$ is equivalent to the $H^{2}(\Omega)$ norm on $W$, that is,

$$
c\|w\|_{H^{2}(\Omega)}^{2} \leq a(w, w) \leq C\|w\|_{H^{2}(\Omega)}^{2} \quad \text { for all } w \in W .
$$

Here and in the sequel, $c$ and $C$ denote generic positive constants.

We introduce the relevant results for the Airy stress function and von Kármán bracket.

Lemma 2.1 [8, Theorem 5.1]. If $w \in H^{2}(\Omega)$ then $\|F(w)\|_{W^{2, \infty}(\Omega)} \leq c\|w\|_{H^{2}(\Omega)}^{2}$. 
Lemma $2.2\left[6\right.$, p. 206]. For $w \in H^{2}(\Omega), \phi \in \widetilde{W}$ and $v \in W$,

$$
\int_{\Omega}[w, \phi] v d \Omega=\int_{\Omega}[w, v] \phi d \Omega .
$$

Lemma $2.3[8, \mathrm{p} .270]$. If $w \in H^{2}(\Omega)$ and $\phi \in W^{2, \infty}(\Omega)$ then

$$
\|[w, \phi]\| \leq c\|w\|_{H^{2}(\Omega)}\|\phi\|_{W^{2, \infty}(\Omega)} .
$$

Lemma 2.4 [3, Proposition 2.4]. If $w_{n} \rightarrow w$ weakly in $H^{2}(\Omega)$ then $F\left(w_{n}\right) \rightarrow F(w)$ weakly in $H_{0}^{2}(\Omega)$.

Definition 2.5. Given $b \in U_{M}, w_{0} \in W$ and $w_{1} \in L^{2}(\Omega), w$ is said to be $a$ weak solution to (1.1)-(1.5) if $w \in C([0, T] ; W) \cap C^{1}\left([0, T] ; L^{2}(\Omega)\right)$ and $w$ satisfies

$$
\begin{gathered}
\int_{0}^{T}\left\{\left\langle w_{t t}, \phi\right\rangle+a(w, \phi)+\left(b w_{t}, \phi\right)\right\} d t=\int_{0}^{T}([w, F(w)], \phi) d t \quad \text { for all } \phi \in W, \\
w(0)=w_{0}, \quad w_{t}(0)=w_{1},
\end{gathered}
$$

where $\langle\cdot, \cdot\rangle$ denotes the duality pairing between $H^{2}(\Omega)$ and its dual space $\left(H^{2}(\Omega)\right)^{\prime}$.

The following regularity result for solutions of (1.1)-(1.5) is given by Cheushov and Lasiecka [6]: given initial data $w_{0} \in H^{4}(\Omega) \cap W$, satisfying the compatibility conditions

$$
\Delta w_{0}+(1-\mu) B_{1} w_{0}=0 \quad \text { on } \Gamma_{1}, \quad \frac{\partial}{\partial v}\left(\Delta w_{0}\right)+(1-\mu) B_{2} w_{0}=0 \quad \text { on } \Gamma_{1},
$$

on the boundary, there exists a unique regular solution $w$ to (1.1)-(1.5) such that $w \in C\left([0, T] ; H^{4}(\Omega) \cap W\right), w_{t} \in C\left([0, T] ; H^{2}(\Omega)\right)$.

Theorem 2.6. For any $w_{0} \in W, w_{1} \in L^{2}(\Omega)$ and $b \in U_{M}$, there exists a unique weak solution $w$ to (1.1)-(1.5) and $w$ satisfies the following estimate:

$$
\|w(t)\|_{H^{2}(\Omega)}^{2}+\left\|w_{t}(t)\right\|^{2} \leq C\left(\left\|w_{0}\right\|_{H^{2}(\Omega)},\left\|w_{1}\right\|\right) \exp (2 M T),
$$

where $C\left(\left\|w_{0}\right\|_{H^{2}(\Omega)},\left\|w_{1}\right\|\right)$ is a constant depending on the values $\left\|w_{0}\right\|_{H^{2}(\Omega)}$ and $\left\|w_{1}\right\|$.

Proof. The proof is similar to that of Theorem 1.1 in the paper by Horn and Lasiecka [9].

\section{Existence of an optimal control}

THEOREM 3.1. There exists an optimal control $b^{*} \in U_{M}$ which minimizes the objective functional $J(w, b)$ over $b$ in $U_{M}$.

Proof. Let $\left\{b^{n}\right\}$ be a minimizing sequence in $U_{M}$, that is,

$$
d \leq J\left(w^{n}, b^{n}\right)=\frac{1}{2} \int_{0}^{T}\left\|w^{n}-z\right\|^{2} d t+\frac{\beta}{2} \int_{\Omega} b^{n}(x, y)^{2} d \Omega \leq d+\frac{1}{n},
$$


where $w^{n}$ is the weak solution of (1.1)-(1.5). Note that $0 \leq d<\infty$. By Theorem 2.6, we know that

$$
\left\|w^{n}(t)\right\|_{H^{2}(\Omega)}^{2}+\left\|w_{t}^{n}(t)\right\|^{2} \leq C\left(\left\|w_{0}\right\|_{H^{2}(\Omega)},\left\|w_{1}\right\|\right) \exp (2 M T) .
$$

From (1.1) and (3.2), we conclude that

$$
\left\|w_{t t}^{n}\right\|_{\left(H^{2}(\Omega)\right)^{\prime}} \leq C\left(\left\|w_{0}\right\|_{H^{2}(\Omega)},\left\|w_{1}\right\|\right) \exp (2 M T) .
$$

From (3.1)-(3.3) we have, along a subsequence,

$$
\begin{aligned}
& w^{n} \rightarrow w^{*} \text { weakly star in } L^{\infty}\left(0, T ; H^{2}(\Omega)\right), \\
& w_{t}^{n} \rightarrow w_{t}^{*} \text { weakly star in } L^{\infty}\left(0, T ; L^{2}(\Omega)\right), \\
& w_{t t}^{n} \rightarrow w_{t t}^{*} \text { weakly star in } L^{\infty}\left(0, T ;\left(H^{2}(\Omega)\right)^{\prime}\right), \\
& b^{n} \rightarrow b^{*} \text { weakly in } L^{2}(\Omega) .
\end{aligned}
$$

Since $U_{M}$ is a closed and convex subset of $L^{\infty}(\Omega) \subset L^{2}(\Omega), U_{M}$ is weakly closed in $L^{2}(\Omega)$. Hence, we obtain $b^{*} \in U_{M} \subset L^{2}(\Omega)$. On the other hand, the fact that $-M \leq b^{n}(x, y) \leq M$ for all $n$ gives $b^{n} \rightarrow b^{* *}$ weakly star in $L^{\infty}(\Omega)$, and hence $b^{n} \rightarrow b^{* *}$ weakly in $L^{2}(\Omega)$. By the uniqueness of the weak limit, we obtain $b^{*}=b^{* *}$ and $b^{*} \in U_{M} \subset L^{\infty}(\Omega)$.

Noting that

$$
w^{n}(t) \rightarrow w^{*}(t) \text { weakly in } H^{2}(\Omega) \text { for almost all } t,
$$

we obtain from Lemma 2.4 that

$$
F\left(w^{n}(t)\right) \rightarrow F\left(w^{*}(t)\right) \text { weakly in } H_{0}^{2}(\Omega) \text { for almost all } t .
$$

Since $\operatorname{dim} \Omega=2$ and (3.8) holds, by Sobolev embedding [1], $F\left(w^{n}(t)\right) \rightarrow F\left(w^{*}(t)\right)$ strongly in $C(\Omega) \subset L^{\infty}(\Omega)$. This and (3.7) imply

$$
\lim _{n \rightarrow \infty} \int_{\Omega}\left[w^{n}(t), \phi\right] d \Omega=\int_{\Omega}\left[w^{*}(t), \phi\right] d \Omega \quad \text { for all } \phi \in W,
$$

that is, $\left[w^{n}(t), \phi\right]$ converges to $\left[w^{*}(t), \phi\right]$ in $L^{1}(\Omega)$. Consequently,

$$
\begin{aligned}
\lim _{n \rightarrow \infty} \int_{\Omega}\left[w^{n}(t), F\left(w^{n}(t)\right)\right] \phi d \Omega & =\lim _{n \rightarrow \infty} \int_{\Omega}\left[w^{n}(t), \phi\right] F\left(w^{n}(t)\right) d \Omega \\
& =\int_{\Omega}\left[w^{*}(t), \phi\right] F\left(w^{*}(t)\right) d \Omega \\
& =\int_{\Omega}\left[w^{*}(t), F\left(w^{*}(t)\right)\right] \phi d \Omega \text { for all } \phi \in W .
\end{aligned}
$$

Now we show that

$$
\lim _{n \rightarrow \infty} \int_{0}^{T}\left(b^{n} w_{t}^{n}(t), \phi\right) d t=\int_{0}^{T}\left(b^{*} w_{t}^{*}(t), \phi\right) d t \quad \text { for all } \phi \in W,
$$


by using the method of Theorem 3.1 in the paper by Bradley and Lenhart [4]. For this, we take $\xi \in C^{1}([0, T])$ and let $\phi(t)=\xi(t) \phi$ for $\phi \in W$. Then

$$
\int_{0}^{T}\left(b^{n} w_{t}^{n}(t), \phi(t)\right) d t=-\int_{0}^{T}\left(b^{n} w^{n}(t), \phi_{t}(t)\right) d t+\left(b^{n} w^{n}(T), \phi(T)\right)-\left(b^{n} w_{0}, \phi(0)\right) .
$$

The convergence results (3.4) and (3.5) and the fact that the embedding $H^{2}(\Omega) \hookrightarrow$ $L^{2}(\Omega)$ is compact yield

$$
w^{n} \rightarrow w^{*} \text { strongly in } C\left([0, T] ; L^{2}(\Omega)\right) .
$$

(This result is proved after some modifications for measure zero by Lions and Magenes [13].) Noting that $\phi \in W \subset C(\Omega)$ and using the convergence results (3.6) and (3.12), we take the limit $n \rightarrow \infty$ in (3.11) and obtain

$$
\begin{aligned}
\lim _{n \rightarrow \infty} \int_{0}^{T}\left(b^{n} w_{t}^{n}(t), \phi(t)\right) d t & =-\int_{0}^{T}\left(b^{*} w^{*}(t), \phi_{t}(t)\right) d t+\left(b^{*} w^{*}(T), \phi(T)\right)-\left(b^{*} w_{0}, \phi(0)\right) \\
& =\int_{0}^{T}\left(b^{*} w_{t}^{*}(t), \phi(t)\right) d t
\end{aligned}
$$

This implies (3.10). Hence, using $w^{n}$ instead of $w$ and taking the limit $n \rightarrow \infty$ in (2.2) and using (3.4)-(3.6), (3.9) and (3.10), we obtain

$$
\int_{0}^{T}\left\{\left\langle w_{t t}^{*}, \phi\right\rangle+a\left(w^{*}, \phi\right)+\left(b^{*} w_{t}^{*}, \phi\right)\right\} d t=\int_{0}^{T}\left(\left[w^{*}, F\left(w^{*}\right)\right], \phi\right) d t \quad \text { for all } \phi \in W .
$$

Now, taking the limit $n \rightarrow \infty$ in (3.1) and noting that the objective functional $J(\cdot, \cdot)$ is weakly lower semicontinuous, we obtain $J\left(w^{*}, b^{*}\right)=d$. This completes the proof.

\section{Characterization of the optimal controls}

Define an operator $G$ by

$$
G f=g \quad \text { if and only if } \quad \Delta^{2} g=f \text { in } \Omega, g=\frac{\partial g}{\partial \nu}=0 \text { on } \Gamma .
$$

One notes that $G[w, w]=-F(w)$ for $w \in H^{2}(\Omega)$.

Proposition 4.1 [8, Theorem 5.1]. The following hold:

(i) The map $(w, u) \mapsto G[w, u]$ is bilinear and bounded from $H^{2}(\Omega) \times H^{2}(\Omega)$ to $H^{3}(\Omega) \cap W^{2, \infty}(\Omega) \cap W^{4,1}(\widetilde{\Omega}), \widetilde{\Omega} \subset \Omega$.

(ii) The map $(w, u, \phi) \mapsto[w, G[u, \phi]]$ is trilinear and bounded from $H^{2}(\Omega) \times$ $H^{2}(\Omega) \times H^{2}(\Omega)$ to $L^{2}(\Omega)$.

Lemma 4.2. For $f \in C^{1}\left([0, T] ; H^{-2}(\Omega)\right)$,

$$
\frac{d}{d t} G(f)=G\left(\frac{d}{d t} f\right)
$$


Proof. Let $f \in C^{1}\left([0, T] ; H^{-2}(\Omega)\right)$ and $g=G(f)$, that is,

$$
\Delta^{2} g(t)=f(t) \text { in } \Omega \quad \text { and } \quad g(t)=\frac{\partial g(t)}{\partial v}=0 \text { on } \Gamma .
$$

Then

$$
\begin{aligned}
\Delta^{2}\left(\lim _{h \rightarrow 0} \frac{1}{h}(g(t+h)-g(t))\right) & =\lim _{h \rightarrow 0} \frac{1}{h} \Delta^{2}(g(t+h)-g(t)) \\
& =\lim _{h \rightarrow 0} \frac{1}{h}(f(t+h)-f(t))=f_{t} .
\end{aligned}
$$

In the first equality we used the fact that the biharmornic operator $\Delta^{2}$ is an isomorphism from $\left\{w \in H^{2}(\Omega): w=\partial w / \partial v=0\right\}$ onto $H^{-2}(\Omega)$ [2]. This implies $G\left(f_{t}\right)=$ $g_{t}=d / d t G(f)$.

Lemma 4.3. The Airy stress function $F(\cdot)$ is Gâteaux differentiable with respect to $w \in H^{2}(\Omega)$ in the direction $u \in H^{2}(\Omega)$. Denoting the Gâteaux derivative of $F(w)$ by $\nabla F(w ; u)$, we have $\nabla F(w ; u)=-2 G[w, u]$.

Proof. Let $w, u \in H^{2}(\Omega)$ and $\epsilon>0$. Then

$$
\begin{aligned}
\lim _{\epsilon \rightarrow 0} \Delta^{2}\left(\frac{F(w+\epsilon u)-F(w)}{\epsilon}\right) & =-\lim _{\epsilon \rightarrow 0} \frac{1}{\epsilon}([w+\epsilon u, w+\epsilon u]-[w, w]) \\
& =-\lim _{\epsilon \rightarrow 0} \frac{1}{\epsilon}[2 w+\epsilon u, \epsilon u]=-2[w, u] .
\end{aligned}
$$

The fact that the biharmornic operator $\Delta^{2}$ is an isomorphism from $\left\{w \in H^{2}(\Omega): w=\right.$ $\partial w / \partial v=0\}$ onto $H^{-2}(\Omega)[2]$ yields

$$
\lim _{\epsilon \rightarrow 0} \Delta^{2}\left(\frac{F(w+\epsilon u)-F(w)}{\epsilon}\right)=\Delta^{2}\left(\lim _{\epsilon \rightarrow 0} \frac{F(w+\epsilon u)-F(w)}{\epsilon}\right) .
$$

Hence we conclude from (4.2) that $F(\cdot)$ is Gâteaux differentiable at $w \in H^{2}(\Omega)$ in the direction $u \in H^{2}(\Omega)$, and the derivative is $\nabla F(w ; u)=-2 G[w, u]$.

LEMMA 4.4. The following hold:

(i) The solution map $b \in U_{M} \mapsto w(b) \in C([0, T] ; W) \cap C^{1}\left([0, T] ; L^{2}(\Omega)\right)$ is continuous.

(ii) The solution map is differentiable in the sense that

$$
\begin{aligned}
& \frac{w(b+\epsilon l)-w(b)}{\epsilon} \rightarrow \psi \text { weakly star in } L^{\infty}(0, T ; W), \\
& \frac{w_{t}(b+\epsilon l)-w_{t}(b)}{\epsilon} \rightarrow \psi_{t} \text { weakly star in } L^{\infty}\left(0, T ; L^{2}(\Omega)\right),
\end{aligned}
$$


as $\epsilon \rightarrow 0$, for any $b, b+\epsilon l \in U_{M}$. Moreover, $\psi$ is a weak solution to the following system:

$$
\left\{\begin{array}{l}
\psi_{t t}+\Delta^{2} \psi+b \psi_{t}+l w_{t}=[\psi, F(w)]-2[w, G[w, \psi]] \quad \text { in } Q \\
\psi(0)=\psi_{t}(0)=0 \quad \text { in } \Omega, \quad \psi=\frac{\partial \psi}{\partial v}=0 \quad \text { on } \Sigma_{0} \\
\Delta \psi+(1-\mu) B_{1} \psi=0 \quad \text { on } \Sigma_{1}, \\
\frac{\partial}{\partial v}(\Delta \psi)+(1-\mu) B_{2} \psi=0 \quad \text { on } \Sigma_{1} .
\end{array}\right.
$$

Proof. Let $\epsilon>0, b, b+\epsilon l \in U_{M}, w^{\epsilon}=w(b+\epsilon l), w=w(b)$ and $z^{\epsilon}=w^{\epsilon}-w$. Then $z^{\epsilon}$ is a weak solution of

$$
\left\{\begin{array}{l}
z_{t t}^{\epsilon}+\Delta^{2} z^{\epsilon}+b z_{t}^{\epsilon}+\epsilon l w_{t}^{\epsilon}=\left[z^{\epsilon}, F\left(w^{\epsilon}\right)\right]+\left[w, F\left(w^{\epsilon}\right)-F(w)\right] \text { in } Q \\
z^{\epsilon}(0)=z_{t}^{\epsilon}(0)=0 \quad \text { in } \Omega, \quad z^{\epsilon}=\frac{\partial z^{\epsilon}}{\partial v}=0 \quad \text { on } \Sigma_{0}, \\
\Delta z^{\epsilon}+(1-\mu) B_{1} z^{\epsilon}=0 \quad \text { on } \Sigma_{1}, \\
\frac{\partial}{\partial v}\left(\Delta z^{\epsilon}\right)+(1-\mu) B_{2} z^{\epsilon}=0 \quad \text { on } \Sigma_{1} .
\end{array}\right.
$$

In what follows, the actual calculations are performed on regular solutions with smooth and compatible initial data, and then final inequalities which are valid for all weak solutions are obtained via density arguments.

Multiplying the first equation of (4.4) by $z_{t}^{\epsilon}$ and integrating the result over $(0, t)$, we obtain

$$
\begin{aligned}
\frac{1}{2}\left\|z_{t}^{\epsilon}\right\|^{2} & +\frac{1}{2} a\left(z^{\epsilon}, z^{\epsilon}\right)-\int_{0}^{t} \int_{\Omega} b(x, y)\left(z_{t}^{\epsilon}\right)^{2} d \Omega d s \\
= & -\epsilon \int_{0}^{t} \int_{\Omega} l(x, y) w_{t}^{\epsilon} z_{t}^{\epsilon} d \Omega d s \\
& +\int_{0}^{t}\left(\left[z^{\epsilon}, F\left(w^{\epsilon}\right)\right], z_{t}^{\epsilon}\right) d s+\int_{0}^{t}\left(\left[w, F\left(w^{\epsilon}\right)-F(w)\right], z_{t}^{\epsilon}\right) d s .
\end{aligned}
$$

Young's inequality and (2.3) give

$$
\begin{aligned}
& \left|\int_{0}^{t} \int_{\Omega} b(x, y)\left(z_{t}^{\epsilon}\right)^{2} d \Omega d s\right| \leq M \int_{0}^{t}\left\|z_{t}^{\epsilon}\right\|^{2} d s \\
& \left|\epsilon \int_{0}^{t} \int_{\Omega} l(x, y) w_{t}^{\epsilon} z_{t}^{\epsilon} d \Omega d s\right| \leq \epsilon C\left(\left\|w_{0}\right\|_{H^{2}(\Omega)},\left\|w_{1}\right\|\right)+\frac{\epsilon}{2} \int_{0}^{t}\left\|z_{t}^{\epsilon}\right\|^{2} d s .
\end{aligned}
$$

Making use of Lemmas 2.1 and 2.2, inequality (2.3) and Young's inequality, we obtain

$$
\begin{aligned}
\left|\int_{0}^{t}\left(\left[z^{\epsilon}, F\left(w^{\epsilon}\right)\right], z_{t}^{\epsilon}\right) d s\right| & \leq C \int_{0}^{t}\left\|z^{\epsilon}\right\|_{H^{2}(\Omega)}\left\|F\left(w^{\epsilon}\right)\right\|_{W^{2, \infty}(\Omega)}\left\|z_{t}^{\epsilon}\right\| d s \\
& \leq C\left(\left\|w_{0}\right\|_{H^{2}(\Omega)},\left\|w_{1}\right\|\right)\left(\int_{0}^{t}\left(\left\|z^{\epsilon}\right\|_{H^{2}(\Omega)}^{2}+\left\|z_{t}^{\epsilon}\right\|^{2}\right) d s\right) .
\end{aligned}
$$


Noting that $F$ is locally Lipschitz and applying (2.3),

$$
\left|\int_{0}^{t}\left(\left[w, F\left(w^{\epsilon}\right)-F(w)\right], z_{t}^{\epsilon}\right) d s\right| \leq C\left(\left\|w_{0}\right\|_{H^{2}(\Omega)},\left\|w_{1}\right\|\right)\left(\int_{0}^{t}\left(\left\|z^{\epsilon}\right\|_{H^{2}(\Omega)}^{2}+\left\|z_{t}^{\epsilon}\right\|^{2}\right) d s\right) .
$$

Applying the four estimates above to (4.5), and also applying Gronwall's inequality, we obtain

$$
\left\|z^{\epsilon}\right\|_{H^{2}(\Omega)}^{2}+\left\|z_{t}^{\epsilon}\right\|^{2} \leq \epsilon C\left(\left\|w_{0}\right\|_{H^{2}(\Omega)},\left\|w_{1}\right\|\right) \exp \left(C\left(\left\|w_{0}\right\|_{H^{2}(\Omega)},\left\|w_{1}\right\|, M\right) T\right)
$$

This gives $\left\|z^{\epsilon}\right\|_{H^{2}(\Omega)}^{2}+\left\|z_{t}^{\epsilon}\right\|^{2} \rightarrow 0$ as $\epsilon \rightarrow 0$. Hence, assertion (i) of the lemma is proved.

We now prove (ii). Let $\psi^{\epsilon}=\left(w^{\epsilon}-w\right) / \epsilon$. Then $\psi^{\epsilon}$ is a weak solution of

$$
\left\{\begin{array}{l}
\psi_{t t}^{\epsilon}+\Delta^{2} \psi^{\epsilon}+b \psi_{t}^{\epsilon}+l w_{t}^{\epsilon}=\left[\psi^{\epsilon}, F\left(w^{\epsilon}\right)\right]+\left[w, \frac{F\left(w^{\epsilon}\right)-F(w)}{\epsilon}\right] \quad \text { in } Q \\
\psi^{\epsilon}(0)=\psi_{t}^{\epsilon}(0)=0 \quad \text { in } \Omega, \quad \psi^{\epsilon}=\frac{\partial \psi^{\epsilon}}{\partial v}=0 \quad \text { on } \Sigma_{0} \\
\Delta \psi^{\epsilon}+(1-\mu) B_{1} \psi^{\epsilon}=0 \quad \text { on } \Sigma_{1}, \\
\frac{\partial}{\partial v}\left(\Delta \psi^{\epsilon}\right)+(1-\mu) B_{2} \psi^{\epsilon}=0 \quad \text { on } \Sigma_{1}
\end{array}\right.
$$

By the same argument as in the above computation, we derive

$$
\left\|\psi^{\epsilon}\right\|_{H^{2}(\Omega)}^{2}+\left\|\psi_{t}^{\epsilon}\right\|^{2} \leq C\left(\left\|w_{0}\right\|_{H^{2}(\Omega)},\left\|w_{1}\right\|\right) \exp \left(C\left(\left\|w_{0}\right\|_{H^{2}(\Omega)},\left\|w_{1}\right\|, M\right) T\right)
$$

This implies, along a subsequence,

$$
\begin{aligned}
& \psi^{\epsilon} \rightarrow \psi \text { weakly star in } L^{\infty}\left(0, T ; H^{2}(\Omega)\right), \\
& \psi_{t}^{\epsilon} \rightarrow \psi_{t} \text { weakly star in } L^{\infty}\left(0, T ; L^{2}(\Omega)\right)
\end{aligned}
$$

Since the solution map is continuous and $F$ is Gâteaux differentiable (see Lemma 4.3), we obtain

$$
\left.\lim _{\epsilon \rightarrow 0}\left\langle\left[w, \frac{F\left(w^{\epsilon}\right)-F(w)}{\epsilon}\right], \phi\right\rangle=\langle[w, \nabla F(w ; \psi)], \phi]\right\rangle=\langle-2 G[w, \psi], \phi\rangle .
$$

By the same argument as in Theorem 3.1 and by using (4.6), we conclude that $\psi$ is a weak solution of (4.3). 


\section{Lemma 4.5. The following hold:}

(i) Let $b \in U_{M}, w \in C^{1}\left([0, T] ; H^{2}(\Omega)\right)$ and $f \in W^{1,2}\left(0, T ; L^{2}(\Omega)\right)$ with $f(0) \in L^{2}(\Omega)$ be given. Then the system

$$
\left\{\begin{array}{l}
p_{t t}+\Delta^{2} p-b p_{t}=[p, F(w)]-2[G[w, p], w]+f \quad \text { in } Q \\
p(0)=p_{t}(0)=0 \quad \text { in } \Omega \\
p=\frac{\partial p}{\partial v}=0 \quad \text { on } \Sigma_{0} \\
\Delta p+(1-\mu) B_{1} p=0 \quad \text { on } \Sigma_{1} \\
\frac{\partial}{\partial v}(\Delta p)+(1-\mu) B_{2} p=0 \quad \text { on } \Sigma_{1}
\end{array}\right.
$$

has a unique regular solution $p \in C\left([0, T] ; H^{4}(\Omega) \cap W\right), p_{t} \in C([0, T] ; W), p_{t t} \in$ $C\left([0, T] ; L^{2}(\Omega)\right)$.

(ii) Let $w \in C\left([0, T] ; H^{2}(\Omega)\right) \cap C^{1}\left([0, T] ; L^{2}(\Omega)\right)$ and $f \in W^{1,2}\left(0, T ; L^{2}(\Omega)\right)$ be given. Then the system (4.7) has a unique weak solution $p \in C([0, T] ; W) \cap$ $C^{1}\left([0, T] ; L^{2}(\Omega)\right)$.

Proof. The proof of the first part of the lemma is standard via the Galerkin method, and the second part is proved by a similar argument to Theorem 1.1 in the paper by Horn and Lasiecka [9].

In order to deduce adjoint equations and obtain necessary conditions for the optimal control, we need the following result, which relies on the fact that $G$ defined in (4.1) is clamped at the boundary.

Lemma 4.6. Let $u, v \in W$ and $w, \phi \in H^{2}(\Omega)$. Then

$$
\int_{\Omega} v[G[w, u], \phi] d \Omega=\int_{\Omega} u[w, G[v, \phi]] d \Omega .
$$

Proof. Let $u, v \in W$ and $w, \phi \in H^{2}(\Omega)$. By the definition of $G$ given in (4.1), it is noted that $G[w, u]$ and $G[v, \phi]$ belong to both $W$ and $\widetilde{W}$. Thus, by Lemma 2.2 and Green's formula,

$$
\begin{aligned}
\int_{\Omega} v[G[w, u], \phi] d \Omega & =\int_{\Omega} G[w, u][v, \phi] d \Omega \\
& =\int_{\Omega} G[w, u] \Delta^{2} G[v, \phi] d \Omega=\int_{\Omega} \Delta^{2} G[w, u] G[v, \phi] d \Omega \\
& =\int_{\Omega}[w, u] G[v, \phi] d \Omega=\int_{\Omega} u[w, G[v, \phi]] d \Omega .
\end{aligned}
$$

We are now in a position to state the characterization of the optimal controls. 
THeOREM 4.7. Let $\left(w^{*}, b^{*}\right)$ be an optimal control pair of the problem (1.6) subject to (1.1)-(1.5). Then $\left(w^{*}, b^{*}\right)$ is characterized by the state and adjoint equations

$$
\begin{aligned}
& \left\{\begin{array}{l}
w_{t t}^{*}+\Delta^{2} w^{*}+b^{*}(x, y) w_{t}^{*}=\left[w^{*}, F\left(w^{*}\right)\right] \quad \text { in } Q, \\
w^{*}(0)=w_{0}, w_{t}^{*}(0)=w_{1} \quad \text { in } \Omega, \\
w^{*}=\frac{\partial w^{*}}{\partial v}=0 \quad \text { on } \Sigma_{0}, \\
\Delta w^{*}+(1-\mu) B_{1} w^{*}=0 \quad \text { on } \Sigma_{1}, \\
\frac{\partial}{\partial v}\left(\Delta w^{*}\right)+(1-\mu) B_{2} w^{*}=0 \quad \text { on } \Sigma_{1},
\end{array}\right. \\
& \left\{\begin{array}{l}
p_{t t}^{*}+\Delta^{2} p^{*}-b^{*} p_{t}^{*}=\left[F\left(w^{*}\right), p^{*}\right]-2\left[G\left[w^{*}, p^{*}\right], w^{*}\right]+w^{*}-z \text { in } Q, \\
p^{*}(T)=p_{t}^{*}(T)=0 \quad \text { in } \Omega, \\
p^{*}=\frac{\partial p^{*}}{\partial v}=0 \quad \text { on } \Sigma_{0}, \\
\Delta p^{*}+(1-\mu) B_{1} p^{*}=0 \quad \text { on } \Sigma_{1}, \\
\frac{\partial}{\partial v}\left(\Delta p^{*}\right)+(1-\mu) B_{2} p^{*}=0 \quad \text { on } \Sigma_{1},
\end{array}\right.
\end{aligned}
$$

where $z$ is the desired evolution given in the cost functional J (see Section 1), and by the relation

$$
b^{*}(x, y)=\max \left\{-M, \min \left\{\frac{1}{\beta} \int_{0}^{T} w_{t}^{*} p^{*} d t, M\right\}\right\} .
$$

Proof. If we set $T-t=t^{\prime}$ then the adjoint equations (4.9) take the form (4.7). So we get the weak solution $p^{*} \in C([0, T] ; W) \cap C^{1}\left([0, T] ; L^{2}(\Omega)\right)$ of (4.9) by Lemma 4.5(ii). We now proceed to characterize the optimal control pair. Let $b^{*}+\epsilon l \in U_{M}, w^{\epsilon}=$ $w\left(b^{*}+\epsilon l\right)$ be the corresponding solution to the state equation. Since $J$ achieves its minimum at $b^{*}$,

$$
\begin{aligned}
0 \leq & \frac{1}{\epsilon}\left(J\left(w^{\epsilon}, b^{*}+\epsilon l\right)-J\left(w^{*}, b^{*}\right)\right) \\
= & \frac{1}{2 \epsilon} \int_{0}^{T}\left\{\left(w^{\epsilon}-w^{*}, w^{\epsilon}-z\right)+\left(w^{*}-z, w^{\epsilon}-w^{*}\right)\right\} d t \\
& +\frac{\beta}{2 \epsilon} \int_{\Omega}\left(2 \epsilon l b^{*}(x, y)+\epsilon^{2} l(x, y)^{2}\right) d \Omega .
\end{aligned}
$$

Taking the limit $\epsilon \rightarrow 0$ in (4.11), we obtain

$$
0 \leq \int_{0}^{T}\left(\psi, w^{*}-z\right) d t+\int_{\Omega} \beta l(x, y) b^{*}(x, y) d \Omega,
$$

where $\psi$ is the solution of (4.3) with $b$ replaced by $b^{*}$.

By Lemma 4.6,

$$
2 \int_{\Omega} \psi\left[G\left[w^{*}, p^{*}\right], w^{*}\right] d \Omega=2 \int_{\Omega}\left[w^{*}, G\left[w^{*}, \psi\right]\right] p^{*} d \Omega .
$$


Applying (4.9) to (4.12) and using (4.13),

$$
\begin{aligned}
\int_{0}^{T}\left\langle\psi, p_{t t}^{*}+\Delta^{2} p^{*}-b^{*} p_{t}^{*}-\left[F\left(w^{*}\right), p^{*}\right]+2\left[G\left[w^{*}, p^{*}\right], w^{*}\right]\right\rangle d t \\
+\beta \int_{\Omega} l(x, y) b^{*}(x, y) d \Omega \\
=\int_{0}^{T}\left\langle\psi_{t t}, p^{*}\right\rangle d t+\int_{0}^{T} a\left(\psi, p^{*}\right) d t+\int_{0}^{T}\left(b^{*} \psi_{t}, p^{*}\right) d t-\int_{0}^{T}\left(p^{*},\left[F\left(w^{*}\right), \psi\right]\right) d t \\
\quad+2 \int_{0}^{T}\left(\left[w^{*}, G\left[w^{*}, \psi\right]\right], p^{*}\right) d t+\beta \int_{\Omega} l(x, y) b^{*}(x, y) d \Omega \\
=-\int_{0}^{T} \int_{\Omega} l(x, y) w_{t}^{*} p^{*} d \Omega d t+\beta \int_{\Omega} l(x, y) b^{*}(x, y) d \Omega \geq 0 .
\end{aligned}
$$

Using a standard control argument based on the choices for the variation $l(x, y)$, we obtain the desired characterization for $b^{*}$, namely equation (4.10).

Now we prove the uniqueness of the optimal control.

THEOREM 4.8. For sufficiently small $T>0$, the solution of the optimality system (4.8)(4.10) is unique.

Proof. Suppose that $\left(w^{*}, b^{*}, p^{*}\right)$ and $(\bar{w}, \bar{b}, \bar{p})$ are two solutions of the optimality system (4.8)-(4.10). It is noted that these two solutions are bounded functions on $\bar{Q}$ (see, for example, (2.3)). Let $\tilde{w}=w^{*}-\bar{w}$ and $\tilde{p}=p^{*}-\bar{p}$. Then $\tilde{w}$ and $\tilde{p}$ are weak solutions of the system

$$
\left\{\begin{array}{l}
\tilde{w}_{t t}+\Delta^{2} \tilde{w}+b^{*} w_{t}^{*}-\bar{b} \bar{w}_{t}=\left[w^{*}, F\left(w^{*}\right)\right]-[\bar{w}, F(\bar{w})] \quad \text { in } Q \\
\tilde{p}_{t t}+\Delta^{2} \tilde{p}-b^{*} p_{t}^{*}+\bar{b} \bar{p}_{t} \\
\quad=\left[p^{*}, F\left(w^{*}\right)\right]-[\bar{p}, F(\bar{w})]-2\left[G\left[w^{*}, p^{*}\right], w^{*}\right] \\
\quad+2[G[\bar{w}, \bar{p}], \bar{w}]+\tilde{w} \quad \text { in } Q \\
\tilde{w}(0)=\tilde{w}_{t}(0)=\tilde{p}(T)=\tilde{p}_{t}(T)=0 \quad \text { in } \Omega \\
\tilde{w}=\frac{\partial \tilde{w}}{\partial v}=0 \quad \text { on } \Sigma_{0} \\
\Delta \tilde{w}+(1-\mu) B_{1} \tilde{w}=0 \quad \text { on } \Sigma_{1} \\
\frac{\partial}{\partial v}(\Delta \tilde{w})+(1-\mu) B_{2} \tilde{w}=0 \quad \text { on } \Sigma_{1} \\
\tilde{p}=\frac{\partial \tilde{p}}{\partial v}=0 \quad \text { on } \Sigma_{0}, \\
\Delta \tilde{p}+(1-\mu) B_{1} \tilde{p}=0 \quad \text { on } \Sigma_{1} \\
\frac{\partial}{\partial v}(\Delta \tilde{p})+(1-\mu) B_{2} \tilde{p}=0 \quad \text { on } \Sigma_{1}
\end{array}\right.
$$

In the sequel, the actual calculations are performed on smooth solutions with smooth and compatible initial data, and then final inequalities which are valid for all weak solutions are obtained via a density argument. Multiplying the first equation of (4.14) 
by $\tilde{w}_{t}$ (respectively, the second equation of (4.14) by $-\tilde{p}_{t}$ ) and integrating by parts over $\Omega \times(0, t)$ (respectively, over $\Omega \times(t, T)$ ), we obtain

$$
\begin{aligned}
\frac{1}{2}\left\|\tilde{w}_{t}\right\|^{2} & +\frac{1}{2} a(\tilde{w}, \tilde{w})+\frac{1}{2}\left\|\tilde{p}_{t}\right\|^{2}+\frac{1}{2} a(\tilde{p}, \tilde{p}) \\
= & -\int_{0}^{t}\left(b^{*} w_{t}^{*}-\bar{b} \bar{w}_{t}, \tilde{w}_{t}\right) d s-\int_{t}^{T}\left(b^{*} p_{t}^{*}-\bar{b} \bar{p}_{t}, \tilde{p}_{t}\right) d s \\
& +\int_{0}^{t}\left(\left[w^{*}, F\left(w^{*}\right)\right]-[\bar{w}, F(\bar{w})], \tilde{w}_{t}\right) d s \\
& -\int_{t}^{T}\left(\left[p^{*}, F\left(w^{*}\right)\right]-[\bar{p}, F(\bar{w})], \tilde{p}_{t}\right) d s \\
& +2 \int_{t}^{T}\left(\left[G\left[w^{*}, p^{*}\right], w^{*}\right]-[G[\bar{w}, \bar{p}], \bar{w}], \tilde{p}_{t}\right) d s-\int_{t}^{T}\left(\tilde{w}, \tilde{p}_{t}\right) d s .
\end{aligned}
$$

Next we estimate the right-hand side of (4.15) term by term. First we estimate $I_{1}=-\int_{0}^{t}\left(b^{*} w_{t}^{*}-\bar{b} \bar{w}_{t}, \tilde{w}_{t}\right) d s$. Making use of $b^{*} \in U_{M}$, the fact that $\bar{w}_{t}$ is bounded and Young's inequality, we obtain

$$
\begin{aligned}
\left|I_{1}\right| & =\left|\int_{0}^{t}\left(b^{*} \tilde{w}_{t}+\left(b^{*}-\bar{b}\right) \bar{w}_{t}, \tilde{w}_{t}\right) d s\right| \\
& \leq M \int_{0}^{t}\left\|\tilde{w}_{t}\right\|^{2} d s+\int_{0}^{t}\left|\left(\left(b^{*}-\bar{b}\right) \bar{w}_{t}, \tilde{w}_{t}\right)\right| d s \\
& \leq\left(M+\frac{c}{2}\right) \int_{0}^{t}\left\|\tilde{w}_{t}\right\|^{2} d s+\frac{c}{2} \int_{\Omega}\left(b^{*}-\bar{b}\right)^{2} d \Omega .
\end{aligned}
$$

For $I_{2}=-\int_{t}^{T}\left(b^{*} p_{t}^{*}-\bar{b} \bar{p}_{t}, \tilde{p}_{t}\right) d s$, similarly we obtain

$$
\left|I_{2}\right| \leq\left(M+\frac{c}{2}\right) \int_{0}^{t}\left\|\tilde{p}_{t}\right\|^{2} d s+\frac{c}{2} \int_{\Omega}\left(b^{*}-\bar{b}\right)^{2} d \Omega .
$$

From (4.10),

$$
\begin{aligned}
\int_{\Omega}\left(b^{*}-\bar{b}\right)^{2} d \Omega & \leq \frac{1}{\beta^{2}} \int_{\Omega}\left|\int_{0}^{T}\left(w_{t}^{*} p^{*}-\bar{w}_{t} \bar{p}\right) d t\right|^{2} d \Omega \\
& \leq \frac{2}{\beta^{2}} \int_{\Omega}\left\{\left(\int_{0}^{T} \tilde{w}_{t} p^{*} d t\right)^{2}+\left(\int_{0}^{T} \bar{w}_{t} \tilde{p}\right)^{2}\right\} d \Omega \\
& \leq c\left\{\int_{0}^{T}\left\|\tilde{w}_{t}\right\|^{2} d t+\int_{0}^{T}\|\tilde{p}\|^{2} d t\right\},
\end{aligned}
$$

where we have used Hölder's inequality and the boundedness of $p^{*}$ and $\bar{w}_{t}$ in the last inequality. 
Next we estimate $I_{3}=\int_{0}^{t}\left(\left[w^{*}, F\left(w^{*}\right)\right]-[\bar{w}, F(\bar{w})], \tilde{w}_{t}\right) d s$. By the fact that $F$ is locally Lipschitz and by Lemma 2.1 ,

$$
\begin{aligned}
\left|I_{3}\right| & \leq\left|\int_{0}^{t}\left(\left[\tilde{w}, F\left(w^{*}\right)\right]+\left[\bar{w}, F\left(w^{*}\right)-F(\bar{w})\right], \tilde{w}_{t}\right) d s\right| \\
& \leq c \int_{0}^{t}\left(\|\tilde{w}\|_{H^{2}(\Omega)}\left\|F\left(w^{*}\right)\right\|_{W^{2, \infty}(\Omega)}\left\|\tilde{w}_{t}\right\|+\|\bar{w}\|_{H^{2}(\Omega)}\|\tilde{w}\|_{H^{2}(\Omega)}\left\|\tilde{w}_{t}\right\|\right) d s \\
& \leq c\left(\int_{0}^{t}\|\tilde{w}\|_{H^{2}(\Omega)}^{2} d s+\int_{0}^{t}\left\|\tilde{w}_{t}\right\|^{2} d s\right),
\end{aligned}
$$

where we have used the boundedness of $w^{*}$ and $\bar{w}$.

For $I_{4}=-\int_{t}^{T}\left(\left[p^{*}, F\left(w^{*}\right)\right]-[\bar{p}, F(\bar{w})], \tilde{p}_{t}\right) d s$, similarly,

$$
\left|I_{4}\right| \leq c\left(\int_{t}^{T}\|\tilde{w}\|_{H^{2}(\Omega)}^{2} d s+\int_{t}^{T}\left\|\tilde{p}_{t}\right\|^{2} d s+\int_{t}^{T}\|\tilde{p}\|_{H^{2}(\Omega)}^{2} d s\right) .
$$

For $I_{5}=2 \int_{t}^{T}\left(\left[G\left[w^{*}, p^{*}\right], w^{*}\right]-[G[\bar{w}, \bar{p}], \bar{w}], \tilde{p}_{t}\right) d s$, by Proposition 4.1 we obtain

$$
\begin{aligned}
\left|I_{5}\right| & =2\left|\int_{t}^{T}\left(\left[G\left[w^{*}, p^{*}\right], \tilde{w}\right]+\left[G\left[w^{*}, \tilde{p}\right], \bar{w}\right]+[G[\tilde{w}, \bar{p}], \bar{w}], \tilde{p}_{t}\right) d s\right| \\
& \leq c_{1} \int_{t}^{T}\|\tilde{w}\|_{H^{2}(\Omega)}\left\|\tilde{p}_{t}\right\| d s+c_{2} \int_{t}^{T}\|\tilde{p}\|_{H^{2}(\Omega)}\left\|\tilde{p}_{t}\right\| d s+c_{3} \int_{t}^{T}\|\tilde{w}\|_{H^{2}(\Omega)}\left\|\tilde{p}_{t}\right\| d s \\
& \leq c\left(\int_{t}^{T}\|\tilde{w}\|_{H^{2}(\Omega)}^{2} d s+\int_{t}^{T}\|\tilde{p}\|_{H^{2}(\Omega)}^{2} d s+\int_{t}^{T}\left\|\tilde{p}_{t}\right\|^{2} d s\right)
\end{aligned}
$$

Finally, for $I_{6}=-\int_{t}^{T}\left(\tilde{w}, \tilde{p}_{t}\right) d s$, Young's inequality yields

$$
\left|I_{6}\right| \leq \frac{1}{2} \int_{t}^{T}\|\tilde{w}\|^{2} d s+\frac{1}{2} \int_{t}^{T}\left\|\tilde{p}_{t}\right\|^{2} d s .
$$

Applying the estimates (4.16)-(4.22) to (4.15), using the Sobolev imbedding $H^{2}(\Omega) \hookrightarrow L^{2}(\Omega)$ and using $(2.1)$, we obtain

$$
\begin{aligned}
& \frac{1}{2}\left\{\left\|\tilde{w}_{t}\right\|^{2}+\|\tilde{w}\|_{H^{2}(\Omega)}^{2}+\left\|\tilde{p}_{t}\right\|^{2}+\|\tilde{p}\|_{H^{2}(\Omega)}^{2}\right\} \\
& \quad \leq \bar{C} \int_{0}^{T}\left(\left\|\tilde{w}_{t}\right\|^{2}+\|\tilde{w}\|_{H^{2}(\Omega)}^{2}+\left\|\tilde{p}_{t}\right\|^{2}+\|\tilde{p}\|_{H^{2}(\Omega)}^{2}\right) d t .
\end{aligned}
$$

Hence, taking $T$ sufficiently small, such that $\bar{C} T<1 / 2$,

$$
\sup _{0 \leq t \leq T}\left\{\left\|\tilde{w}_{t}\right\|^{2}+\|\tilde{w}\|_{H^{2}(\Omega)}^{2}+\left\|\tilde{p}_{t}\right\|^{2}+\|\tilde{p}\|_{H^{2}(\Omega)}^{2}\right\} \leq 0,
$$

which completes the proof. 


\section{References}

[1] R. Adams, Sobolev spaces (Academic Press, New York, 1975).

[2] A. Boutet de Monvel and I. Chueshov, "Uniqueness theorem for weak solutions of von Kármán evolution equations", J. Math. Anal. Appl. 221 (1998) 419-429; doi:10.1006/jmaa.1997.5681.

[3] M. E. Bradley and I. Lasiecka, "Global decay rates for the solutions to a von Kármán plate without geometric conditions", J. Math. Anal. Appl. 181 (1994) 254-276; doi:10.1006/jmaa.1994.1019.

[4] M. E. Bradley and S. Lenhart, "Bilinear spatial control of the velocity term in a Kirchhoff plate equation", Electron. J. Differential Equations 2001 (2001) 1-15; http://emis.maths.adelaide.edu.au/journals/EJDE/Volumes/2001/27/abstr.html.

[5] M. E. Bradley, S. Lenhart and J. Yong, "Bilinear optimal control of the velocity term in a Kirchhoff plate equation", J. Math. Anal. Appl. 238 (1999) 451-467; doi:10.1006/jmaa.1999.6524.

[6] I. Chueshov and I. Lasiecka, "Inertial manifolds for von Kármán plate equations", Appl. Math. Optim. 46 (2002) 179-206; doi:10.1007/s00245-002-0741-7.

[7] I. Chueshov and I. Lasiecka, "Global attractors for von Kármán evolutions with a nonlinear boundary dissipation", J. Differential Equations 198 (2004) 196-231; doi:10.1016/j.jde.2003.08.008.

[8] A. Favini, M. A. Horn, I. Lasiecka and D. Tataru, "Global existence, uniqueness and regularity of solutions to a von Kármán system with nonlinear boundary dissipation", Differential Integral Equations 9 (1996) 267-294; http://projecteuclid.org/euclid.die/1367603346.

[9] M. A. Horn and I. Lasiecka, "Uniform decay of weak solutions to a von Kármán plate with nonlinear boundary dissipation", Differential Intergral Equations 7 (1994) 885-908.

[10] J. Lagnese, Boundary stabilization of thin plates (SIAM, Philadelphia, 1989).

[11] I. Lasiecka, "Finite dimensionality and compactness of attractors for von Kármán equations with nonlinear dissipation", NoDEA Nonlinear Differential Equations Appl. 6 (1999) 437-472; doi:10.1007/s000300050012.

[12] J. L. Lions, Quelques méthodes de résolution des problèmes aux limites non linéaires (Dunod, Paris, 1969).

[13] J. L. Lions and E. Magenes, Problèmes aux Limites non homogènes et applications (Dunod, Paris, 1968).

[14] J. Y. Park and S. H. Park, "Uniform decay for a von Kármán plate equation with a boundary memory condition", Math. Methods Appl. Sci. 28 (2005) 2225-2240; doi:10.1002/mma.663.

[15] J.-P. Puel and M. Tucsnak, "Boundary stabilization for the von Kármán equations", SIAM. J. Control Optim. 33 (1995) 255-273; doi:10.1137/S0363012992228350. 